\title{
Nanoscale Quantum Laboratories
}

\section{G. Hörmandinger}

\author{
Imperial College of Science, Technology and Medicine, London
}

The idea of quantum confinement has been around in condensed matter physics for several decades. The usual method of trapping electrons in a localized region in space is by means of semiconductor heterostructure devices, using layered structures and lithographic techniques (structures can now be made which are only a few tens of nanometres in size). A completely different route has been opened up recently by advances in scanning tunnelling microscopy (STM) in combination with various techniques of surface preparation. STM uses an extremely sharp probing tip which is brought close to the surface of a sample, allowing electrons to tunnel between the sample and the tip (Fig. 1a). The technique essentially measures the local density of states (LDOS) of the electrons, i.e., the energy-resolved electron density, at the position of the apex of the tip. The structures which confine the electrons are located at the surface of a crystal. They are built using molecular beam epitaxy, thermal treatment, or even the manipulation into the required geometric patterns of individual adsorbed atoms by the STM tip.

In the case of semiconductor heterostructures, the main interest concerns transport properties through a device. In contrast, STM allows the observation in real-space of the electron wave functions (or at least their moduli squared). The only restriction for the operation of a STM is that the sample be electrically conducting.

\section{Electrons on Metallic Surfaces}

At first sight, the idea of confining an electron on a metallic surface sounds rather hopeless, given that metals are conductors. What makes it possible is the periodicity of the crystal, giving rise to Bloch's theorem [1]: upon changing position in real space by a lattice vector $\boldsymbol{R}$, the wave function is multiplied by exp (ikR), where $\boldsymbol{k}$ is a wave vector within the symmetric unit cell in reciprocal space, the so-called first Brillouin zone. States with different $\boldsymbol{k}$ vectors are orthogonal to each other. The Bloch states form energy bands separated by band gaps in which electrons cannot reside. This is not to say that there are no Bloch states in these gaps, but that they have complex $\boldsymbol{k}$ vectors which cannot be normalized. Introducing a

Günter Hörmandinger was educated at the Technical University, Vienna, where he received his Ph.D. in 1991. He is currently working as a postdoctoral research assistant in the Physics Department, Imperial College, London, on the theory of STM in connection with adsorbates, thin layers and surface states, but will shortly switch to energy policy and analysis.

Fig. 1 - Schematic illustration of the quantum confinement of electrons at surfaces. From the top: a) a schematic view of a tunnelling junction in the scanning tunnelling microscope. Electrons tunnel from the tip into the bulk of the sample. If there is a surface state, some of the electrons may end up travelling along the surface.

b) The surface band structure (energy as a function of the parallel k-vector) typical of the noble metal (111) surface. The s-band of the metal gives rise to the continuum of bulk states (shaded area). A surface state (dashed line) resides in the projected bulk band gap.

c) A cross-section through the tunnelling junction in a) showing the surface state on the sample side. The complete Bloch wave function in the $x$-direction leads an exponentially decaying envelope function (dashed lines). The state also decays exponentially into the barrier, but owing to the finite width some of the electrons can tunnel through.

d) Surface standing waves arise in STM owing to interference. In the configuration
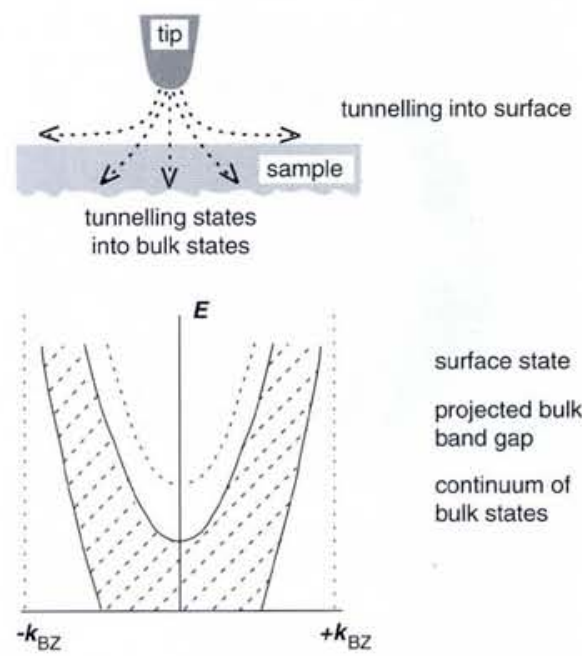

illustrated here, an electron tunnelling from the tip can either end up in a surface state propagating to the left, or it can propagate to the right, be reflected off the adsorbate, and then propagate to the left. The two paths interfere with one another either constructively or destructively at the tip position, leading to a position-dependent tunnelling current in the surface state.

surface can make the gap states allowed, by cutting off their divergent part and replacing it with a function that drops to zero in the vacuum outside the surface (Fig. 1c). Such a surface state can exist if there is at least a partial gap in the projection of the bulk band structure perpendicular to the surface. The surface state cannot couple to any of the bulk states since all of these have different parallel $\boldsymbol{k}$-vectors.

In order to prevent an electron from entering the bulk, one therefore needs a single crystal with a precisely oriented surface. Secondly, the Fermi surfaces of $\mathrm{Cu}$, $\mathrm{Ag}$ and $\mathrm{Au}$ show a neck pointing in the (111) direction. This causes a projected band gap on the close-packed (111) surfaces. It supports a surface state with an almost perfectly parabolic dispersion relation thus making it an excellent free-particle-like model system (Fig. 1b). An electron in such a state will run along the surface, much like a two-dimensional plane wave.

\section{Standing Waves}

Scattered waves and interference fringes are generated when a wave hits an obstacle, causing standing waves in the LDOS. In 1991, Davis et al. observed oscillations in the STM conductance near a step on the $\mathrm{Au}(111)$ surface and explained them in terms of standing waves of the surface state [2]
(Fig. 1d). But it still raised eyebrows last year when two groups based in different IBM laboratories came up with stunning pictures of these standing waves on the $\mathrm{Cu}(111)[3]$ and the $\mathrm{Au}(111)$ [4] surfaces.

States with different energies and therefore with different wavelengths could be probed by changing the bias voltage of the STM. Both groups managed to measure the dispersion relation $E(k)$ of the surface state in this way. Normally, the measurement of $E(k)$ requires techniques such as angleresolved photoemission for occupied states, or inverse photemission for unoccupied states, which are selective both in energy and in $k$-space. STM, in contrast, samples a range of energies and $\boldsymbol{k}$-vectors. The standing waves observed near a step edge are not those of one individual surface state, but belong to the LDOS which is a sum over all available states. The finite bias voltage means that states with different energies are mixed in, so a full understanding of the observed wavelengths requires some further analysis [5].

\section{Building Cages}

Interesting finite-size effects occur if the surface state can be trapped between scatterers of some sort. The problem is how to engineer these scattering objects on the nanometre scale in order to create confining 

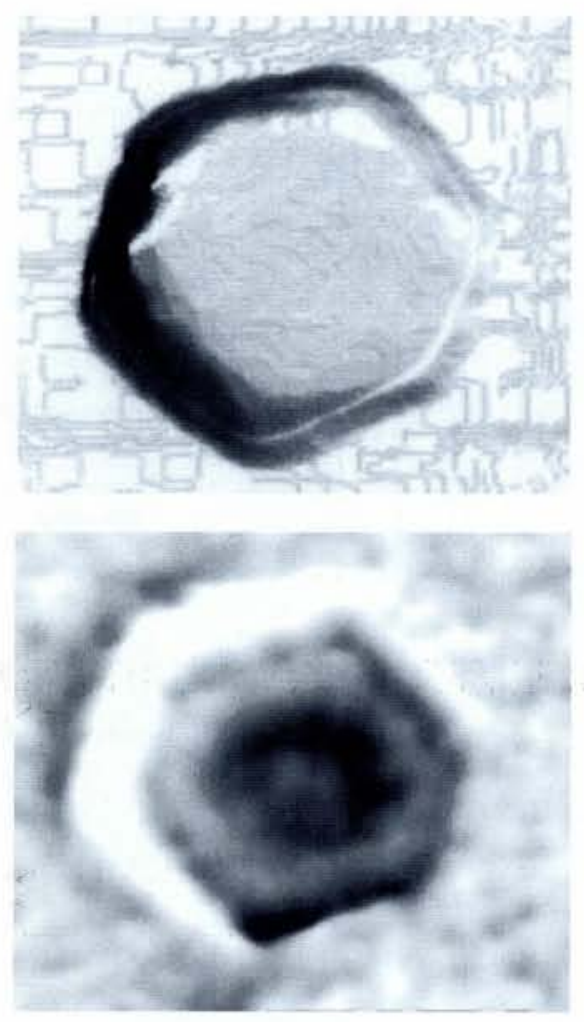

Fig. 2 - a, upper) A topographic STM image of a $4.5 \mathrm{~nm}$-wide monatomic $\mathrm{Ag}(111)$ island on a Ag surface. $b$, lower) A differential conductivity (di/dV) STM image taken a room temperature of the same island at a sample voltage of $-0.01 \mathrm{~V}$. It shows a clear standing wave pattern encompassing the island that generates a large confining potential barrier. (From [6])

structures. Phaedon Avouris and In-Whan Lyo at IBM Yorktown Heights exploited monatomic steps as their waveguides [6]. By growing a stepped $\mathrm{Au}(111)$ surface on mica using controlled epitaxy they produced $3.6 \mathrm{~nm}$-wide terraces which act as quantum wires for the surface state, causing asymmetric peaks in the conductivity spectrum owing to the one-dimensional character of the states. They also looked at a monatomic step bent into a closed ring - that is, an island - on $\mathrm{Ag}(111)$. They observed, even at room temperature, the confined zerodimensional states within a $4.5 \mathrm{~nm}$ in diameter island (see Fig. 2).

The group of Don Eigler at IBM Almaden employ a different technology to erect boundaries for the surface-state electrons. They have worked on the manipulation of individual adsorbed atoms with a STM tip ever since they wrote the name of their company with letters that were compsed of only five $\mathrm{Xe}$ atoms [7]. This method was used to build a circular "quantum corral" made of $48 \mathrm{Fe}$ atoms on a $\mathrm{Cu}(111)$ surface [8] (Fig. 3). The diameter of the corral is $14.26 \mathrm{~nm}$, using images taken at liquidhelium temperatures. The binding energies of the waves of the corral's interior local density of states were determined using the spectroscopic differential conductivity (di/dV) mode of the STM. They were found to be well explained by assuming a freeelectron gas with circular, hard-wall confine-

Fig. 3-A scanning tunnelling microscope image of a circular "quantum corral" made from $48 \mathrm{Fe}$ atoms arranged in a $14.26 \mathrm{~nm}$ in diameter ring on a $\mathrm{Cu}(111)$ surface. Note the strong modulation of the energy-resolved electron density within the ring. Illustration courtesy of IBM Research Division, Almaden Research Center, USA.

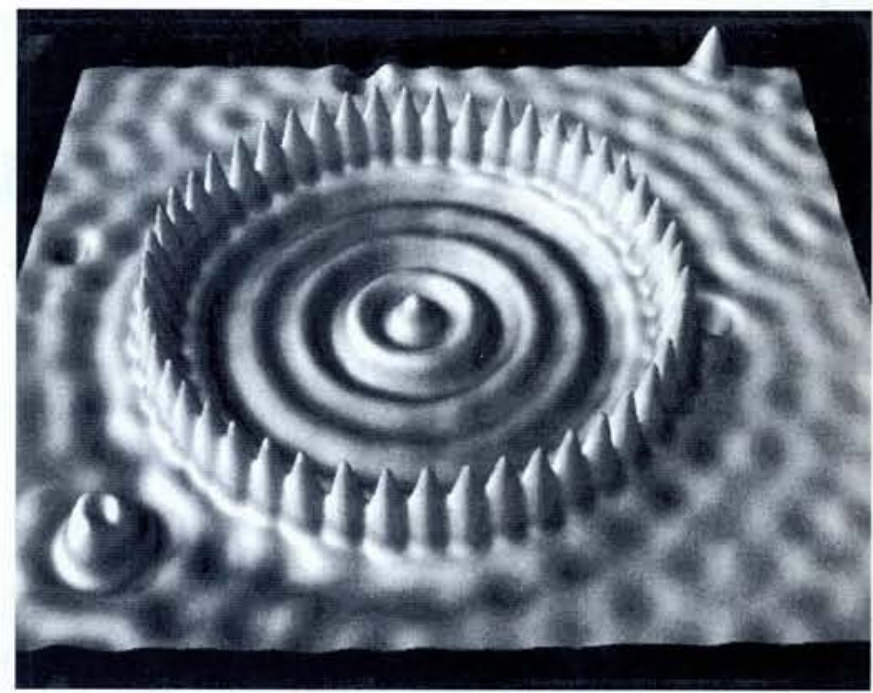

ment. A number of other corrals have also been built from atoms of different elements.

\section{The Nanoscale Quantum Laboratory}

The technological advances in nanostructures allow the creation of all sorts of boundary conditions for a two-dimensional electron gas. Possible applications abound. For example, with appropriate boundary conditions, a two-dimensional electron gas may exhibit quantum chaos. Fig. 4 shows a stadium shape built by the Almaden group [9] - a shape which is one of the candidates for chaotic behaviour.

The scattering of electrons at an adatom (an adsorbed atom) is of considerable interest in itself. Adatoms may interact via the surface state, leading to interesting questions concerning, for instance, the magnetic coupling. There is also the question of the extent to which a surface state couples to the bulk at an adatom or at a step.

This last effect is one of the major difficulties on the route to an ideal, two-dimensional model system. Any obstacle located in the path of a surface-state electron couples the state to a continuum of bulk states. Heller et al. [9] find that the $\mathrm{Fe}$ atoms in their stadium shape can be described by s-wave scatterers with an absorbing character. The

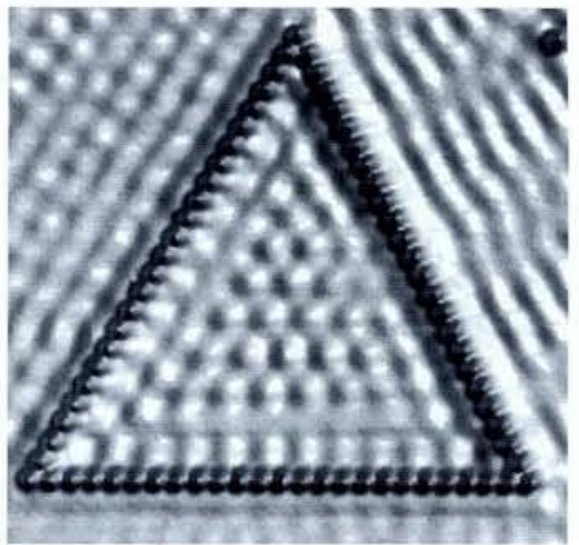

Fig. $4-A$ stadium made of $76 \mathrm{Fe}$ atoms, with dimensions $14.1 \times 28.5 \mathrm{~nm}^{2}$. (From [9]) reflection rate at a row of these scatterers is only $25 \%$, while another $25 \%$ is transmitted into the surface state on the other side and $50 \%$ is "absorbed" (i.e., scattered into the bulk). Similar conclusions were reached by Avouris and Lyo for step edges. These empirical findings are backed up by theoretical work on the quantum corral done by Simon Crampin (Cambridge) and Mike Boon and John Inglesfield (Nijmegen) [10], as well as calculations made at Imperial College for a variety of added rows and step edges. The quality factor $Q$ of a cavity gives a measure of the lifetime of resonances in the cavity. It determines how many times a wave interferes with itself before being absorbed. Quantum chaos requires interference over a long period of time, and therefore a high $Q$. In each of these analyses, scattering into the bulk is the dominant loss mechanism, making the quality factor $Q$ of the cavities rather low. As Heller et al. put it: "In an acoustic analogy, the corral is therefore a rather quiet chamber".

So full-blown quantum chaos in surfacestate electrons may elude observation after all. But the technology is still developing very rapidly, and the applications so far have already outgrown all expectations.

[1] See e.g., Ashcroft N.W. \& Mermin N.D., Solid State Physics (Saunders College Publishing, 1976).

[2] Davis L.C. et al., Phys. Rev. B 43 (1991) 3821.

[3] Crommie M.F., Lutz C.P. \& Eigler D.M. Nature 363 (1993) 524.

[4] Hasegawa Y. \& Avouris Ph., Phys. Rev. Lett. 71 (1993) 1071.

[5] Hörmandinger G., Phys. Rev. B 49 (1994) 13897.

[6] Avouris Ph. \& In-Whan Lyo, Science 264 (1994) 942.

[7] Eigler D.M. \& Schweizer E.K., Nature 344 (1990) 524

[8] M.F. Crommie, C.P. Lutz \& Eigler D.M., Science 262 (1993) 218.

[9] Crommie M.F., Lutz C.P. \& Eigler D.M., Nature 369 (1994) 464.

[10] Crampin S., Boon M.H. \& Inglesfield J.E., Phys. Rev. Lett. (submitted). 